\title{
Charge-exchange nuclear reactions at NSCL using radioactive beams: Development of the LENDA low energy neutron detector array
}

\author{
C. Caesar ${ }^{1,2, t}$, Sam M. Austin ${ }^{1,3}$, D. Bazin ${ }^{1}$, J. M. Deaven ${ }^{1,3,4}$, C. J. Guess ${ }^{1,3,4}$, \\ G. W. Hitt ${ }^{1,3,4}$, R. T. Meharchand ${ }^{1,3,4}$, D. T. Nguyen ${ }^{1,4}$, G. Perdikakis ${ }^{1,3, *}$, \\ Y. Shimbara ${ }^{5}$, K. Thorne ${ }^{6}$, R. G. T. Zegers ${ }^{1,3,4}$ \\ ${ }^{I}$ National Superconducting Cyclotron Laboratory, Michigan State University, East Lansing, \\ Michigan, 48824-1321, USA \\ ${ }^{2}$ Johannes Gutenberg Universität, D-55099 Mainz, Germany \\ ${ }^{3}$ Joint Institute for Nuclear Astrophysics, Michigan State University, East Lansing, \\ Michigan 48824, USA \\ ${ }^{4}$ Department of Physics and Astronomy, Michigan State University, East Lansing, \\ Michigan 48824, USA \\ ${ }^{5}$ Graduate School of Science and Technology, Niigata University, Niigata 950-2181, Japan \\ ${ }^{6}$ Michigan Technological University, Houghton, Michigan 49931-1295,USA \\ E-mail: caesarenscl.msu.edu
}

\begin{abstract}
Charge-exchange reactions on stable nuclei have been used frequently to study the spin-isospin response on nuclei, which is, for example, crucial for estimating weak-transition rates in late stellar evolution. Because a large fraction of the relevant nuclei in such scenarios are unstable, there is a strong need to expand charge-exchange studies to unstable nuclei, which requires that experiments must be carried out in inverse kinematics. A promising probe for experiments in the $\Delta \mathrm{T}_{\mathrm{z}}=-1$ direction is the $(p, n)$ reaction. The "Low Energy Neutron Detector Array" (LENDA) is currently under development at the NSCL (National Superconducting Cyclotron Laboratory). The array will consist of 24 plastic scintillator bars with dimensions of $300 \times 45 \times 25 \mathrm{~mm}$. The neutron energy is measured using the time-of-flight technique. Position sensitivity along the bar is achieved by combining the timing and pulse height signals. In this work results of measurements using prototype modules and results of simulations are presented.
\end{abstract}

10th Symposium on Nuclei in the Cosmos

Mackinac Island, Michigan, USA

27 July - 1 August, 200

\begin{tabular}{ll}
\hline$*$ & Speaker \\
$\mathrm{t}$ & This work was supported by the US NSF, grants PHY-0216783 (JINA) and PHY-0606007
\end{tabular}




\section{Introduction}

Charge-exchange reactions at intermediate energies (100-200 MeV/u) have been used extensively for nuclear structure studies on stable nuclei [1]. In such reactions, a proton in the target nucleus is exchanged with a neutron in the projectile or vice versa. The isospin transfer is $\Delta \mathrm{T}=1$ (isovector). Gamow-Teller (GT) transitions $(\Delta \mathrm{T}=1, \Delta \mathrm{S}=1, \Delta \mathrm{L}=0)$, in particular, have received strong interest. That is because the initial and final states of a $\beta$-decaying nucleus can also be populated through GT transitions in charge-exchange reactions. Since the GT strength is to a good approximation proportional to the charge-exchange cross section in the limit of vanishing momentum transfer [2], charge-exchange reactions have become the preferred tool to extract GT strengths in energy regions inaccessible in $\beta$-decay experiments due to $\mathrm{Q}_{\beta}$-value restrictions.

Weak transitions play an important role in late stellar evolution. Models of supernovae and neutron-star crusts require realistic weak-transition rates [3], which are usually calculated in shell- or mean-field models. These models have to be tested against experimental data, and charge-exchange experiments on stable nuclei have been employed extensively to provide such data. However, since a large fraction of the nuclei that play a role in the stellar scenarios are unstable, there is a strong need to expand the experiments to nuclei beyond the valley of stability.

The lack of charge-exchange studies on unstable nuclei is due to the fact that these experiments must be carried out in inverse kinematics, which makes them very challenging. Measurements have to be performed at small center-of-mass scattering angles to fulfill the condition of small momentum transfer. As a consequence, the probe recoil has low kinetic energy (0-3 MeV). As an example, the kinematics for the $p\left({ }^{38} \mathrm{~K},{ }^{38} \mathrm{Ca}\right) n$ reaction is shown in Figure 1.

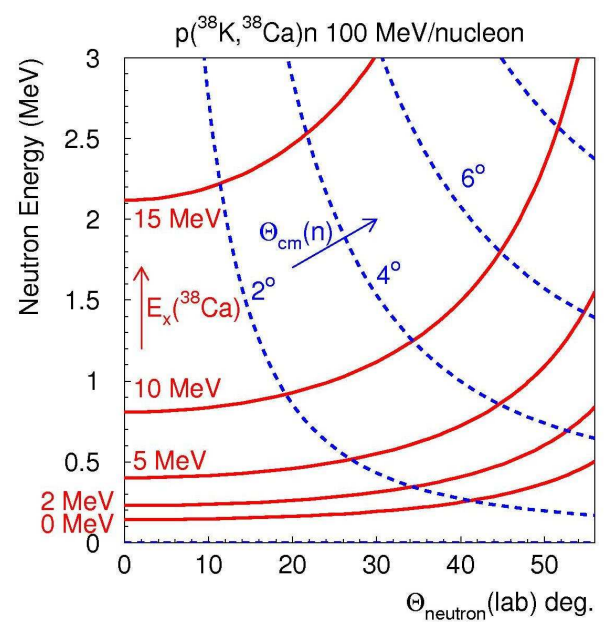

Figure 1. (color online) Kinematical diagram for the $p\left({ }^{38} \mathrm{~K},{ }^{38} \mathrm{Ca}\right) n$ reaction, at a beam energy of 100 $\mathrm{MeV} / \mathrm{u}$. The dashed (blue) lines represent center-of-mass scattering angles as a function of neutron laboratory angle (horizontal axis) and neutron kinetic energy (vertical axis), while the solid (red) lines correspond to excitation energies of the ${ }^{38} \mathrm{Ca}$ residual.

It is challenging to detect such low energy recoil particles. However, this is crucial for cleanly identifying the charge-exchange events and to reconstruct relevant kinematic observables such as the excitation energy and the scattering angle. To achieve the required resolution, it is necessary that the recoil can escape from the target with minimal energy loss and straggling. One of the most promising probes for studying charge-exchange reactions on unstable nuclei in 
the $\Delta \mathrm{T}_{\mathrm{z}}=-1$ direction is the $(p, n)$ reaction in inverse kinematics. Since the recoil is a neutron, it can escape unperturbed even from thick targets. This is important because experiments with unstable beams are performed at modest beam intensities. The experimental challenge is to detect the low-energy neutron with high efficiency and sufficient energy and position resolution. To achieve a resolution comparable to stable-beams $(p, n)$ experiments $(\sim 1 \mathrm{MeV}$ in excitation energy and a few degrees in scattering angle) at low neutron recoil energies, a specialized neutron detector has to be used, having the resolution requirements listed below. For this purpose, the Low Energy Neutron Detector Array is constructed at NSCL.

\section{The Low Energy Neutron Detector Array}

To achieve sufficient energy and angular resolutions for $(p, n)$ experiments in inverse kinematics, LENDA is designed to have timing and position resolution for low energy neutrons better than $1 \mathrm{~ns}$ and $5 \mathrm{~cm}$, respectively. The final array will consist of $24300 \mathrm{~mm} \times 45 \mathrm{~mm} \times 25 \mathrm{~mm}$ BC-408 plastic scintillators. On both long ends of each scintillator bar, gain-selected Hamamatsu H6410 photomultiplier tube (PMT) assemblies are attached. The design of the bars is similar to the one used by Aoi et al. [4] or Beyer et al. [5]. The PMTs are directly glued to the scintillator bar using optically clear epoxy. The scintillators are polished to enhance internal reflection and are wrapped with three layers. For the innermost layer, a filter paper with high diffusive reflectance is used to increase light collection. The second layer is made of aluminum foil. The outer layer is black insulating tape. The second and third layers mainly serve to ensure light-tightness. At present, prototype studies are ongoing using 3 bars. Most tests are conducted using a ${ }^{252} \mathrm{Cf}$ source, which emits correlated neutrons and photons from fission. A NE-213 liquid scintillator serves as a trigger detector. The source is placed $\sim 5 \mathrm{~cm}$ away from this detector and $1 \mathrm{~m}$ away from the prototype LENDA modules. Pulse-shape discrimination is utilized to select photons in the trigger counter, which thus provides a clean trigger signal. In Figure 2, the response of the LENDA array to events from the fission source is shown.
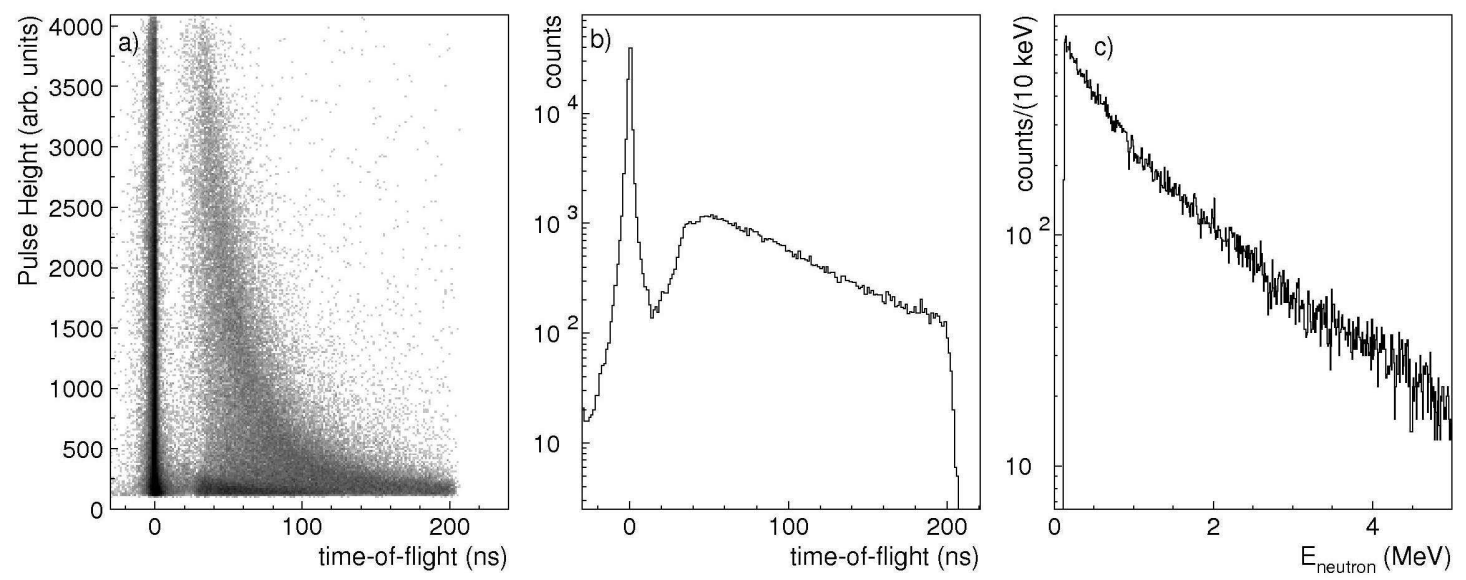

Figure 2. Spectra taken with a ${ }^{252} \mathrm{Cf}$ source placed $1 \mathrm{~m}$ away from the detector. a) PMT pulse-height versus time of flight $b$ ) projection of a) on the horizontal axis representing the time-of-flight $c$ ) reconstructed neutron energy spectrum from the neutron time-of-flight signal.

The events at $\mathrm{t}=0$ in Figure 2a) correspond to $\gamma-\gamma$ coincidences in the trigger detector and LENDA. These events that represent the time-of-flight of photons from the ${ }^{252} \mathrm{Cf}$ source to LENDA, serve as an absolute time reference. Such events are clearly separated in time-of-flight 
from the $n-\gamma$ coincidences. Events of the latter type display a correlation between time-of-flight and PMT pulse-height. The width of the $\gamma-\gamma$ peak in the time-of-flight spectrum (set at $t=0$ in Figures 2a) and 2b)) provides a measure for the time-of-flight resolution. It is about $1 \mathrm{~ns}$ (FWHM), but that value is largely due to the response of the trigger counter. Tests using a ${ }^{22} \mathrm{Na}$ source (only correlated $\gamma-\gamma$ pairs) and a different trigger detector, have shown that the timing resolution of LENDA is better than 600 ps. From the time-of-flight spectrum, the neutron kinetic energy can be reconstructed, as shown in Figure 2c). The lower-limit in the spectrum is $130 \mathrm{keV}$. This value does not define the threshold of the LENDA array, but was set by the range of the Time-to-Digital Converter. The true threshold is just below $100 \mathrm{keV}$.

\section{Light transport simulations and Position calibration}

The response of the scintillator depends strongly on the transmission properties of the propagating medium and the properties of the reflecting boundaries and wrapping materials. To study such effects, the code GUIDE7 [5] was used. The geometry and physical properties, such as reflectances and indices of refraction of the system are input. The output of the simulation allows for tracking of each photon through the scintillator, and for determination of the intensity of the scintillation light arriving at each PMT. The intensity decreases along the flight path due to attenuation in the scintillator material and due to light loss at the scintillator boundaries. GUIDE7 has been modified to reproduce more accurately the combined effects of the wrapping material and total internal reflection. In addition, to compare the output with real data, further processing was applied to take into account the response of the PMT and the CFD (Constant Fraction Discriminator). Figure 3a) shows the results for the distribution of the photon transit times to the PMT at three different hit points along the LENDA bar. A clear correlation between position of hit point and time distribution exists. The index of refraction of the BC-408 scintillator being 1.58, a photon would need $\sim 1.6 \mathrm{~ns}$ to travel the $30 \mathrm{~cm}$ length of the bar in a straight path. However most of the photons undergo a large number of reflections and thus have a considerably longer transit time. This effect is clearly observed in the simulated time distributions of Figure 3a).

By combining the signals from both phtototubes attached to each bar the hit position of each event can be determined. The time information and the integrated charge from each PMT provide a measure of that position. Using a ${ }^{22} \mathrm{Na}$ source in the test setup, a series of measurements to determine the hit position sensitivity has been performed. The source was placed $10 \mathrm{~cm}$ away from the detector and a collimator was used to irradiate a $1.6 \mathrm{~cm}$ wide area of the detector (hit area), at several positions along the scintillator bar. For each event in a measurement, the time difference,

$$
\Delta t=t_{1}-t_{2},
$$

between both PMTs and the corresponding center of gravity of the QDC (charge (Q) to Digital Converter) signals $\mathrm{q}_{1}$ and $\mathrm{q}_{2}(\operatorname{cog} \mathrm{Q})$,

$$
\operatorname{cog} Q=\frac{q_{1}-q_{2}}{q_{1}+q_{2}},
$$

were re-constructed. For each hit area, a gaussian fit was used to determine these quantities. As expected from the simulation, a clear correlation is found experimentaly between position and time difference. The good correspondence between simulated and experimental data is presented in Figure $3 \mathrm{~b})$. Using this linear correlation a position resolution of $(6.2 \pm 0.1) \mathrm{cm}$ in 
FWHM (Full Width at Half Maximum) is achieved. A position resolution of $(8.4 \pm 0.4) \mathrm{cm}$ is achieved by using the cogQ method (not shown). However, by combining the $\Delta \mathrm{t}$ and $\operatorname{cog} \mathrm{Q}$ information, a hit position resolution of $(5.2 \pm 0.3) \mathrm{cm}$ is achieved. That result is consistent with the aforementioned requirements for the array.
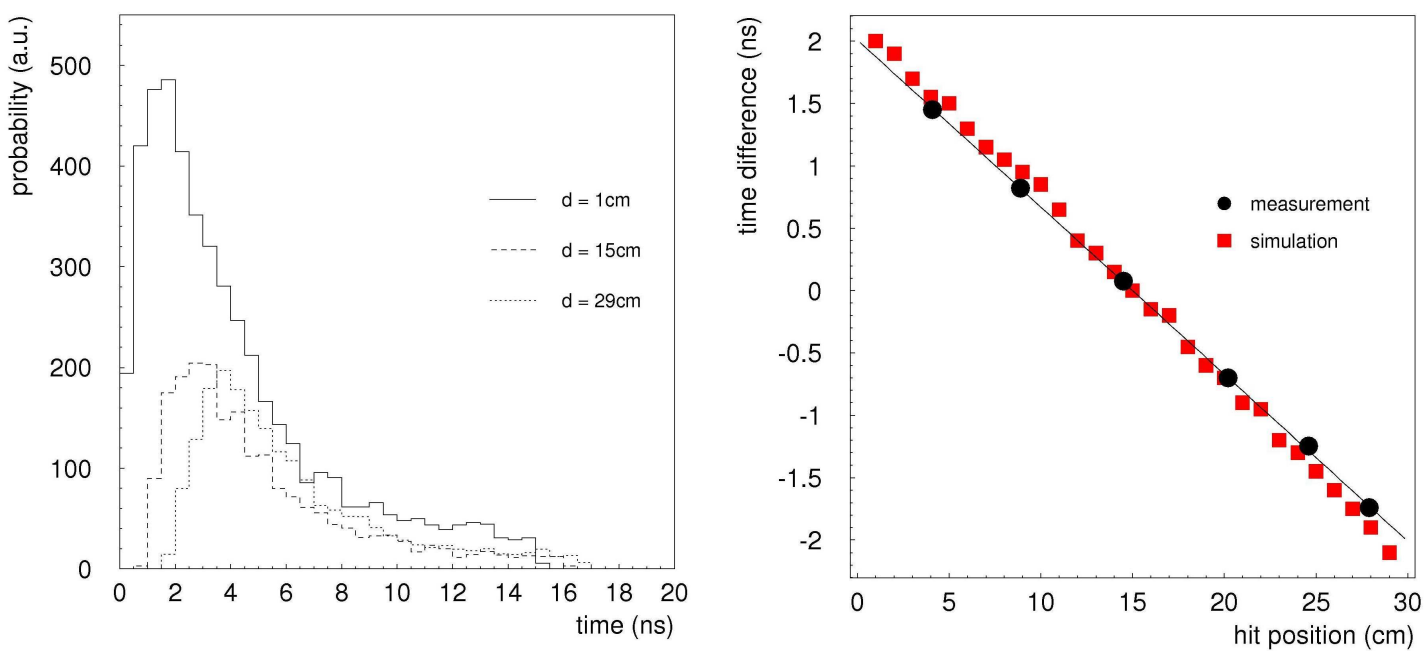

Figure 3. a) Simulation plot of the time distribution of photons arriving at one of the PMTs, for three different hit positions. b) (color online) time difference vs. hit position. (range: 1-29 cm). The (black) circles are the measurement the (red) quadrats are the simulation

\section{Conclusion}

A Low Energy Neutron Detector Array is currently being developed by the NSCL chargeexchange group. It will be used to perform $(p, n)$ charge-exchange reactions in inverse kinematics with unstable beams. The response of the prototype to neutrons was studied using a ${ }^{252} \mathrm{Cf}$ source. Gamma rays from a ${ }^{22} \mathrm{Na}$ source have been used to determine its position resolution. Measurements were complemented by simulations of the detector's optical response. The results verify that the required time and position resolution for the charge-exchange experiments can be achieved. An efficiency measurement for low energy neutrons will be performed in the near future.

\section{References}

[1] F. Osterfeld, Nuclear spin and isospin excitations, Rev. Mod. Phys. 64, 491 (1992)

[2] T. N. Tadeucci et al., The $(p, n)$ reaction as a probe of beta decay strength, Nucl. Phys. A469, 125 (1987)

[3] K. Langanke and G. Martinez-Pinedo, Nuclear weak-interaction processes in stars, Rev. Mod. Phys. 75, 819 (2003)

[4] N. Aoi et al, Anomaly of $N=8$ shell closure in neutron-rich Be and B isotopes studied via delayed neutron emitting ${ }^{14}$ Be $\beta$ decay, Phys. Rev. c 66, 014301 (2002)

[5] R. Beyer et al. „Proton-recoil detectors for time-of-flight measurements of neutrons with kinetic energies from some tens of keV to a few MeV“ Nucl. Instr. and Meth. A 575, (2007), 449 [6] T. Massam, GUIDE7 a general program for evaluating the properties of scintillation and Cerenkov counter optical systems, Tech. Rep. 76-21, CERN, Geneva, 1976. 